\title{
Significance of the high-latitude geomagnetic index AES-80: comparison with the $\mathrm{PC}$ index
}

\author{
P. Ballatore ${ }^{1,2}$ and C. G. Maclennan ${ }^{1}$ \\ ${ }^{1}$ Bell Laboratories, Lucent Technologies, Murray Hill, NJ 07974, U.S.A. \\ ${ }^{2}$ LPCE/CNRS, Orleans, 45071, France
}

(Received October 7, 1998; Revised June 23, 1999; Accepted June 24, 1999)

\begin{abstract}
A new index AES-80 was derived in order to provide a measure of the geomagnetic activity at high southern latitudes (Ballatore et al., 1998). The comparison between the southern hemisphere AES-80 with the northern hemisphere PC index is reported here for the period May 1994-April 1995. It is found that during southward IMF the two parameters record the geomagnetic disturbances rather similarly. In contrast, during northward IMF, only AES-80 is capable of recording geomagnetic activity occurring in the nominal polar cap region. In agreement with this finding, a slightly higher correlation coefficient is found between AES-80 and PC during southward IMF than during the northward IMF condition. Although at any time the two indices reflect opposite seasons, the overall correlations found between the two indices are significant, independent of the specific UT interval considered. The UT modulation (which should possibly be taken into account in the use of the indices) has been calculated for the AES-80 and the PC index. The results show that, for the specific period considered, the PC index dependence on UT is more significant than that of the AES-80 parameter.
\end{abstract}

\section{Introduction}

The auroral electrojet index (AE) is derived from simultaneous geomagnetic measurements at twelve longitudinally spaced stations located at northern auroral latitudes and provides information about the intensity of the currents flowing in the auroral ionosphere (Davis and Sugiura, 1966). This $\mathrm{AE}$ index is calculated as the difference of its two components $\mathrm{AU}$ and $\mathrm{AL}$, which represent, respectively, the maximum eastward and westward currents in the region above the considered stations (Davis and Sugiura, 1966).

An index considered for latitudes higher than the nominal auroral zone is the PC index, which is derived from magnetic data at a single ground-based, near-pole station (Troshichev et al., 1988). Although derived from ground based geomagnetic data, in a statistical sense the PC index has been considered as a measure of the merging interplanetary electric field at the magnetopause (Vennerstrøm et al., 1991).

Derivation of AE-like indices for latitudes higher than the auroral zone, the polar cap, was previously done on a trial basis using geomagnetic data from four Antarctic stations between about $-77^{\circ} \mathrm{CGM}$ latitude and about $-83.8^{\circ} \mathrm{CGM}$ latitude (Saroso et al., 1992; Saroso, 1993).

The most recently developed index for high latitudes is the AES-80 index, which is derived using geomagnetic measurements at five stations longitudinally spaced at $-80^{\circ} \mathrm{CGM}$ latitude. This index provides information about the intensity of currents flowing in the southern polar-cap ionosphere (Ballatore et al., 1998; referred to as paper I in the following). AES-80 is calculated similarly to AE. In particular, it is ob-

Copy right $(\mathrm{C})$ The Society of Geomagnetism and Earth, Planetary and Space Sciences (SGEPSS); The Seismological Society of Japan; The Volcanological Society of Japan; The Geodetic Society of Japan; The Japanese Society for Planetary Sciences. tained as the difference between its components AUS-80 (at each UT, the maximum positive variation at the considered stations) and ALS-80 (at each UT, the maximum negative variation at the stations), which can be related respectively to the maximum eastward and westward currents above the considered stations (see paper I for details). We stress that the AES-80 index does not monitor the auroral oval current system since it is derived at about $-80^{\circ} \mathrm{CGM}$ latitude.

Geomagnetic activity in the polar cap region is due to different phenomena which can be very localized, such as polar cap substorms (e.g., Weatherwax et al., 1997), or which can affect the entire nominal polar cap region, such as the poleward shrink of the auroral oval (Ballatore et al., 1998). In either of these cases it is useful to have a regional index, like AES-80, which collects geomagnetic information from as many stations as possible that are located in the polar cap. The geomagnetic information given by AES-80 is expected to be different than the cross-polar cap current information shown by the PC index, which is a single station indicator.

In the present paper, a comparison between the southern AES-80 index and the northern PC index is discussed.

\section{Data Analysis and Experimental Observations}

A comparison between the PC index calculated at Thule station (e.g., Vennerstrøm et al., 1991) and the AES-80 index was performed. The time period considered is May 1994April 1995, consistent with data availability. The PC index data used are the 15-min data available at the WWW address of the Danish Meteorological Institute; the average values of these data over 1-hour periods are used in the analysis. The AES-80 data are 1-hour averages of the index that are derived at 1 -min resolution from the geomagnetic $\mathrm{H}$-component from 


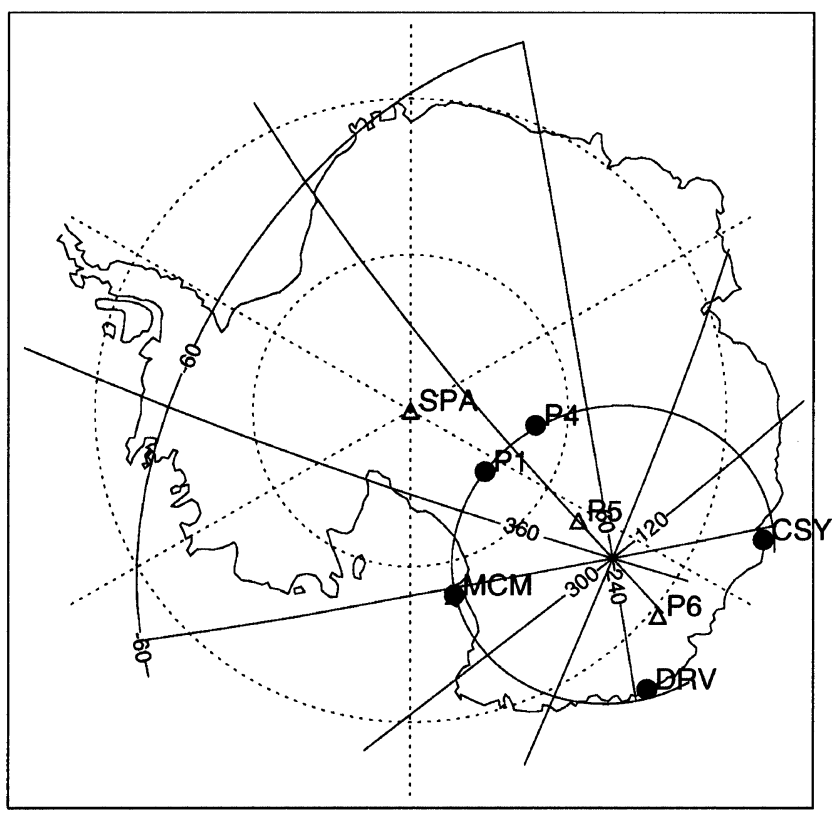

Fig. 1. Location of the five stations, shown as solid circles, used in the derivation of AES-80 index.

the five Antarctic stations shown in Fig. 1. These are all located at about $-80^{\circ} \mathrm{CGM}$ latitude (paper I).

Several specific UT periods have been studied in order to elucidate some differences and similarities between the PC, AES-80 and AE indices in terms of their dependence on interplanetary quantities. The specific period 12-13 October 1994 is shown in Fig. 2. The interplanetary data are 1-hour averages from the IMP-8 satellite, from the OMNI database at the NSSDC WWW address [http://nssdc.ngdc.nasa.gov]; the regular data gaps are due to the satellite orbit configuration. In Fig. 2, an increase of both PC and AES- 80 begins at about 18 UT on October 12, which corresponds to a negative IMF $B_{z}$ (of about $-6 \mathrm{nT}$ ). At the same time, an increase of geomagnetic activity is monitored by the AE index (seventh panel from the top Fig. 2).

During the period 00-08 UT on October 13, the behaviour of PC and AES- 80 is different; in fact the PC index decreases rapidly to values close to zero while AES- 80 remains relatively high. The behaviour of the AE index, in this specific case, is similar to the $\mathrm{PC}$ index: the $\mathrm{AE}$ index also rapidly decreases at time $00 \mathrm{UT}$ (October 13) and remains low for the entire period until about $08 \mathrm{UT}$. The value of AE is, on average, lower than AES-80 during this period, in particular, at $01 \mathrm{UT}$ (AE is 69 and AES- 80 is 127) and at $05 \mathrm{UT}$ (AE is 124 and AES-80 is 153). This period corresponds to northward IMF (about $6 \mathrm{nT}$ ), with a large variation in the $\mathrm{B}_{\mathrm{z}}$ component that decreases to slightly negative values (minimum at 04 UT) and increases again to rather strong northward values. At the same time, no important variations are evident in the solar wind speed; however the ion density is definitely higher than in the following time interval.

In Fig. 3 a second specific UT period (4-5 December 1994) is illustrated. It can be seen that, in the time interval 20-24 UT on December 5, the IMF is strongly northward and AES-
80 is greater than the $\mathrm{AE}$ index, while the $\mathrm{PC}$ index is close to zero.

The scatter plots and correlation coefficients between PC and AES-80, ALS-80, AUS-80 are shown in Fig. 4(a), for northward IMF, and in Fig. 4(b), for southward IMF. The entire year of data, for all UTs, at all seasons, is included here. Results show that the coefficient of the correlation between the PC index and the AES-80 index is slightly higher during southward IMF. Moreover we note that the best correlations are found for AES- 80 vs. PC and ALS- 80 vs. PC (at a confidence level well above 99.9\%), while for AUS-80 vs. PC (top panels) the correlation is definitely lower (although still statistically significant).

In order to examine the possible time dependence of the correlations illustrated in Fig. 4, the correlation coefficients between PC and AES-80, ALS-80 and AUS-80 have been calculated separately for each one-hour UT period for the entire year. The results obtained are shown in Fig. 5, where the number of data points for each UT hour is shown at the top of the panel. No significant time dependence is evident for the correlations of PC with AES-80, while slightly higher dependence of correlation on UT is present for ALS-80. On the contrary, a large decrease is shown in the time interval 08-10 UT for the correlation coefficient of PC with the AUS80 .

The average and the median of the AES- 80 , of the PC and of the AE index, have been calculated for separate UT hours for the common period when the AE is presently available, that is May-December 1994. The AE data are 1-hour averages of the "provisional" index, and this means that they are derived using data from only 11 of the standard 12 stations. However these provisional values can be considered a good approximation of the standard auroral electrojet indices, particularly at the time resolution of 1-hour (instead of the 1-min at which these indices are derived) that we consider here. Results are shown in Fig. 6. It is shown that the variations of AES-80 at different UT are not more significant than the PC ones. In fact $\mathrm{PC}$ varies of about 0.7 and its average value is of the order of 1 and AES-80 varies of about 60 and its average value is of the order of 100 . Moreover the increasing of AES-80 at 21-24 UT is similar to the increase of AE index at the same range of time. The difference between the average value and the median is more significant for the $\mathrm{AE}$ index than for the other two parameters.

We have, finally, analyzed the relationship of PC and AES80 with the IMF $B_{z}$ component considering the entire period May 1994-April 1995. The correlations are not significant for positive IMF $B_{z}$, while for negative $B_{z}$ we found a correlation coefficient 0.4 (with number of data points 2182) for AES-80 and 0.37 (with number of data points 2514) for the PC index. Both correlation coefficients are above the $99.9 \%$ confidence level. For purposes of comparison, also the correlation between $\mathrm{AE}$ and IMF $\mathrm{B}_{\mathrm{Z}}$ has been calculated for the period for which, at present, $\mathrm{AE}$ is available (May-December 1994). The correlation coefficient is not significant for positive IMF $B_{z}$, while it is 0.39 (with number of data points 1148) for negative $B_{z}$. We also studied the relationships of these data with the IMF $\mathrm{B}_{\mathrm{y}}$ component (not shown); no significant correlations were found. 


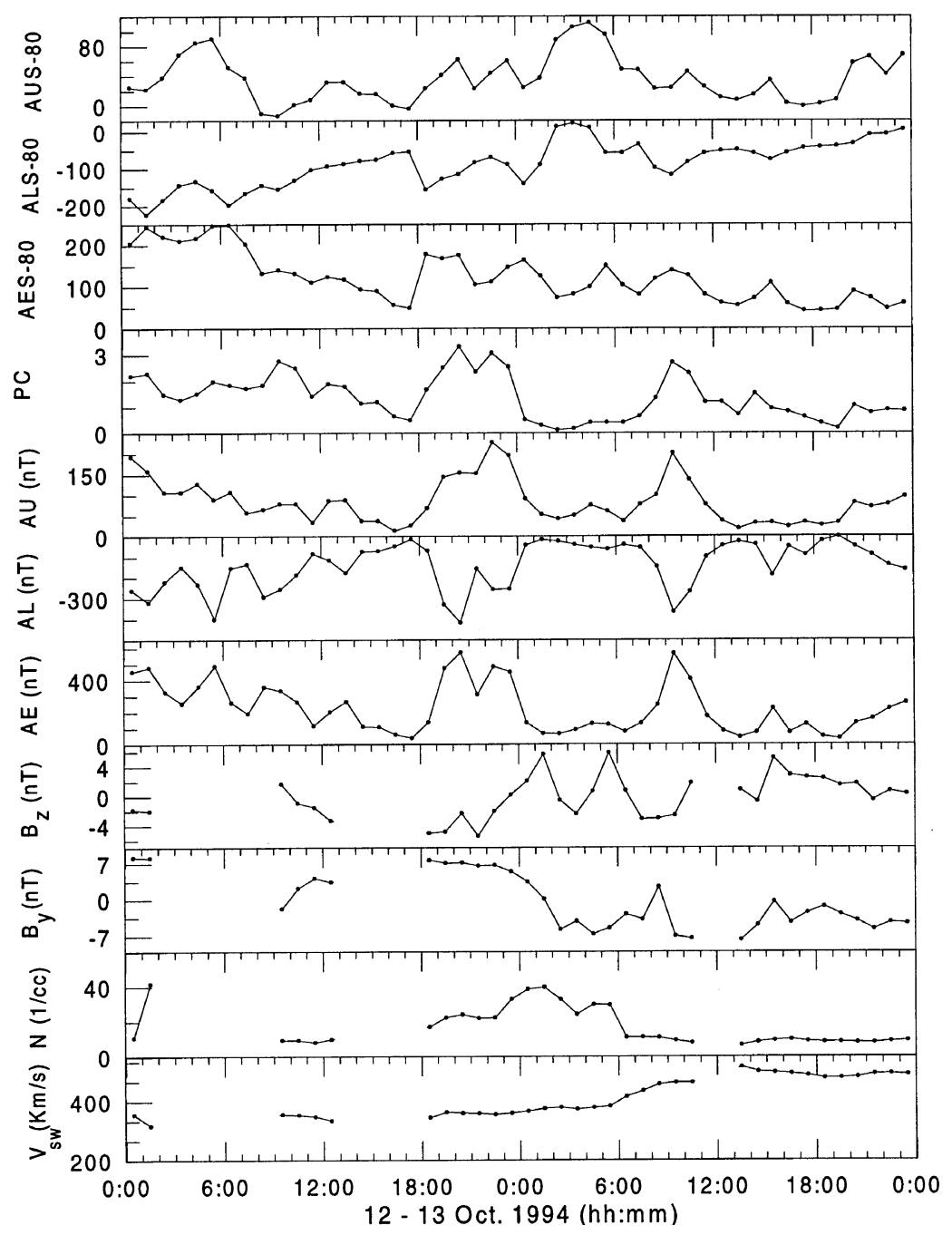

Fig. 2. Plot vs. UT of (from the bottom to the top) solar wind speed $\left(V_{s w}\right)$, ion density $(N), I M F B_{y}$ and $B_{Z}$ components, $A E, A L$ and $A U$ indices, $P C$ index, AES-80, ALS-80 and AUS-80 index for 12-13 October 1994.

\section{Discussion and Conclusion}

The exemplary event in Fig. 2 shows that, when negative IMF $B_{z}$ occurs and drives a geomagnetic disturbance, it is recorded by all three indices, PC, AES- 80 and $\mathrm{AE}$ (as shown by their enhancement at about 20 UT on October 12, 1994).

A different behaviour emerges for AES- 80 with respect to $\mathrm{PC}$ and AE during the period $00-08$ UT on 13 October. This period is not quiet with respect to the interplanetary conditions. The solar wind speed is rather constant, however the ion density is relatively high. Moreover the IMF, which is mostly northward, varies significantly and IMF $B_{z}$ rapidly changes from relatively high positive values to slightly negative and back again to highly positive. Also, a variation of more than $10 \mathrm{nT}$ is observed for the IMF $\mathrm{B}_{\mathrm{y}}$, which goes from positive to negative values. This kind of interplanetary configuration is expected to drive geomagnetic activity in the polar cap region, which is connected most directly to the interplanetary space due to the geomagnetic field line configuration. Geomagnetic activity is in fact observed by AES-80. However, it is not monitored by the PC and AE indices. In this case, the absence of geomagnetic activity seen by the PC index does not indicate a quiet IMF condition or the absence of polar cap currents, but can be attributed to the fact that this index is derived from data from only one ground based station. In fact, since one station cannot collect information from the entire polar cap, the PC index cannot always successfully detect geomagnetic disturbances which last less than the one day required for the measuring station to traverse the entire polar cap. The fact that AE does not monitor geomagnetic activity in the present example can be explained in terms of the poleward shrinking of the auroral oval that typically characterizes northward IMF conditions. This explanation is confirmed by the fact that at times, during this time interval, AES-80 monitors a higher geomagnetic disturbance level than AE.

A second example of the different behaviour of AES80 and PC during northward IMF is given in Fig. 3, when strongly positive IMF $B_{z}$ occurs in the time interval between 21 and 24 UT on December 51994.

Previous results show the capability of polar cap AE-like indices to monitor geomagnetic polar cap activity in the case of southward IMF and confirm the usefulness of polar cap AE-like indices, such as AES-80, for monitoring ionospheric currents associated with the northward IMF (Saroso 


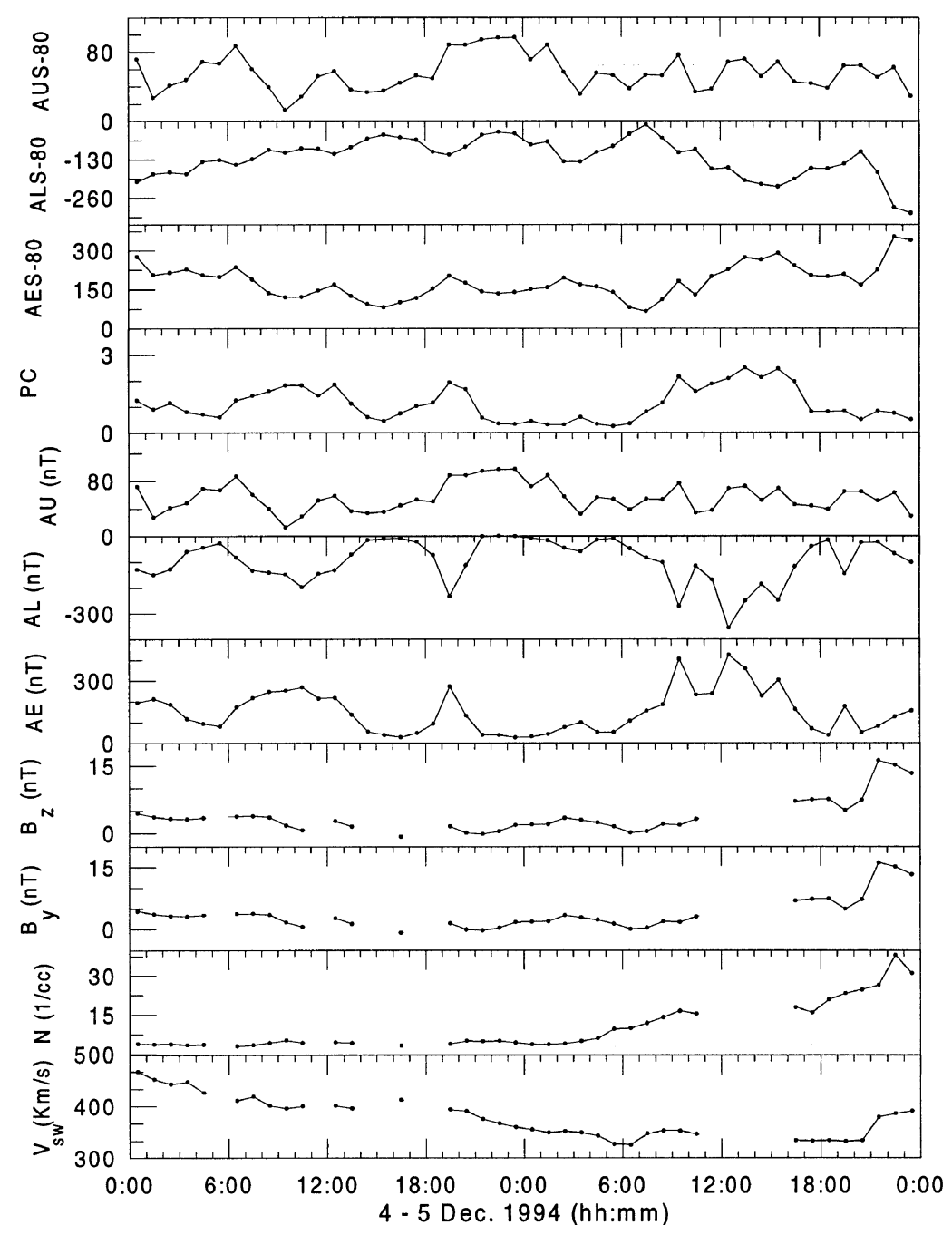

Fig. 3. Similar to Fig. 2 for 4-5 December 1994.

et al., 1992).

The higher agreement between AES-80 and PC, during southward IMF with respect to the northward one, could be confirmed, on a statistical basis, by slightly higher correlation found between the two indices during negative IMF $B_{z}$, as illustrated in Fig. 4.

In particular it is worth noting that the two indices PC and AES-80 monitor the geomagnetic activity in the northern and southern polar cap regions, respectively. Although the seasonal difference implies different ionospheric conductance in the two polar caps, particularly in winter and summer, a significant correlation (well above $99.9 \%$ confidence level) is always found between the two indices over the entire year. The correlations of the two components ALS-80 and AUS80 of the AES- 80 index with PC have been considered separately. This implies the consideration, separately, of the eastward and westward components of the ionospheric currents at about $-80^{\circ} \mathrm{CGM}$ latitude (Paper I). The results show that, although the correlations of ALS- 80 and AES- 80 with the PC index are similar (the difference in correlation coefficient is about 0.1 ), AUS-80 has a definitely lower correlation (although still above the $99.9 \%$ confidence level). Considering that PC and AUS-80 are in opposite hemispheres (therefore, at each UT of comparison, they are at opposite seasons), our result is in agreement with a stronger effect of the seasonal variability in the ionospheric conductance on the eastward (as opposed to westward) components of currents. This has been found particularly at auroral latitudes (e.g., by Allen and Kroehl, 1975).

It is of interest to consider the finding that the significance of the correlation between AES-80 and PC is not strongly dependent on the UT time interval considered. In fact, the correlations between PC and AES-80 for separate UT intervals (as illustrated in Fig. 5) show that the coefficients vary by at most about 0.2 , being always above a minimum value of 0.6 (well above the $99.9 \%$ confidence level). This is not so for the two components ALS-80 and AUS-80. In particular, a large decrease for the AUS- 80 correlation with PC is found in the interval 08-10 UT. A similar decrease in correlation was previously found for AU vs. AUS-80 during the period May-June 1994 (paper I). In that case, the UT time of minimum correlation was found to be between 10 and 12 UT; this was explained in terms of the seasonal dependence of ionospheric conductance, which is also appropriate in the present 


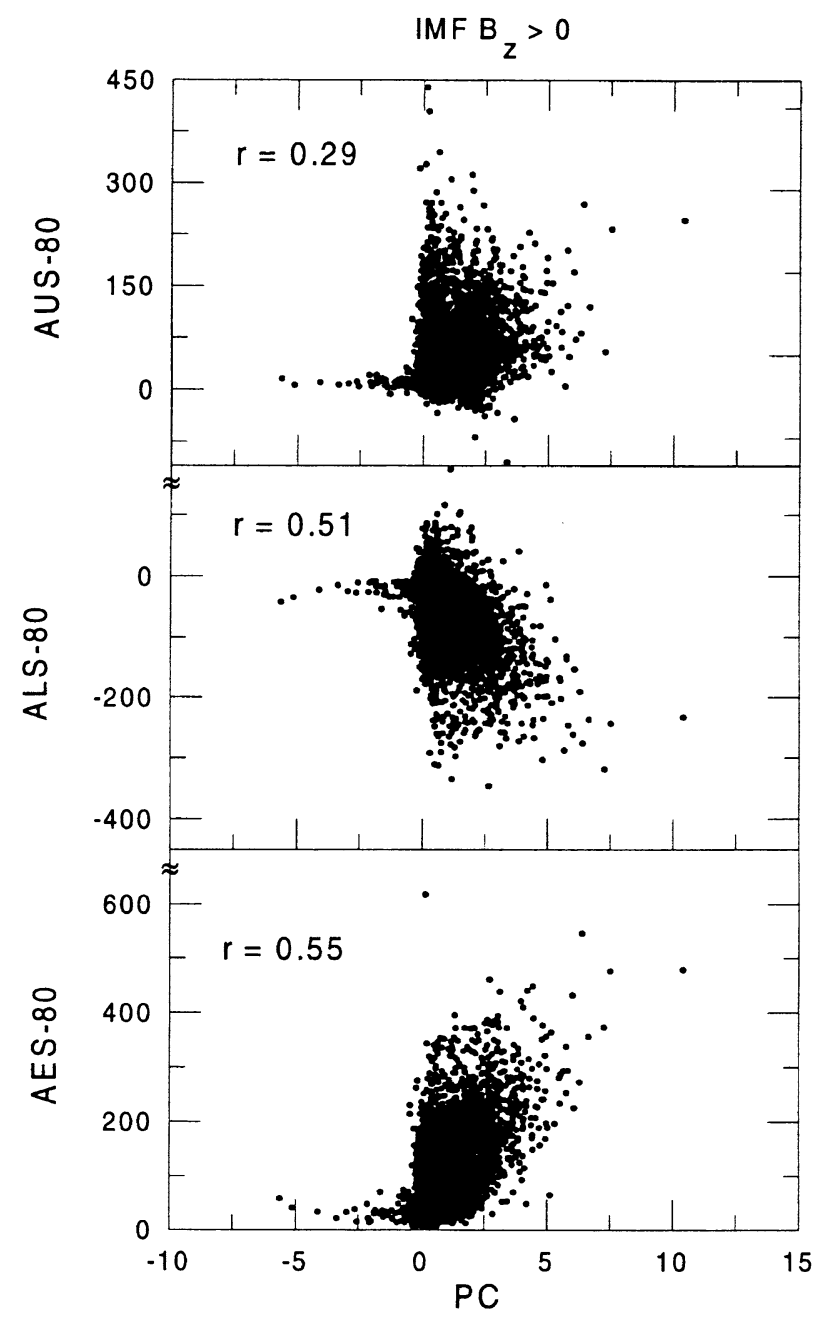

(a)

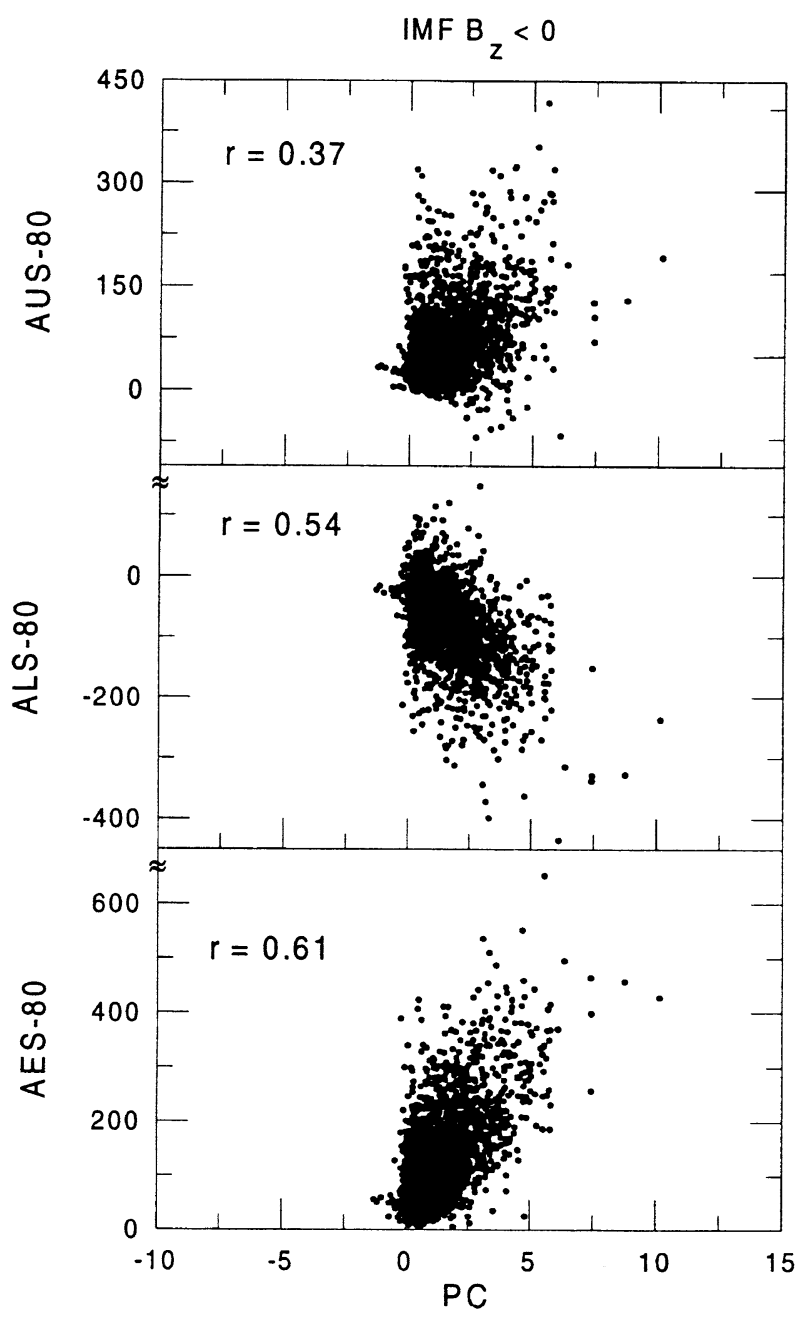

(b)

Fig. 4. (a) Scatter plots of the PC index vs. AUS-80 (top panel), ALS-80 (middle panel) and AES-80 (bottom panel) during northward IMF-the number of data points in each scatter plot is 5484; (b) similar to Figure (a) but for southward IMF-the number of data points in each scatter plot is 2185 .

case because, as previously noted, PC and AUS-80 refer to opposite hemispheres at each time. In these terms, the specific UT period of minimum correlation is not meaningful and we note that it does not correspond to the UT period of minimum correlation between AU and AUS- 80 shown in paper I. In addition, we speculate that the low correlation in the interval 08-10 UT that we find between PC and AUS-80 could be related to the fact that the PC index is formed from only one station. It could be that in this interval ( $\sim 6-8$ MLT at Thule) the PC index fails to see eastward currents that are measured by at least one of the five stations that are used for AUS-80.

In order to show the overall effectiveness of the parameter AES-80, it is important to check its UT variation. In fact, in principle, if a geomagnetic index depends on UT, the average UT variation should be subtracted in using it. Therefore, the average values and the median values of AES- 80 and PC are represented in Fig. 6 for the period May-December 1994. A higher level of AES-80 is found between 21 and 7 UT; however, this depends on the specific period considered. In particular the increase of average activity for $21-24$ UT is rather similar to what is found for the AE index in the top panel of Fig. 6. The variation of PC seems rather regular with higher level during about half day. In addition the level of the maximum UT variation of PC is not smaller than the one for the AES-80. This might indicate a relatively good effectiveness of the parameter AES-80 compared with the $\mathrm{PC}$ index in order to indicate a global polar cap geomagnetic activity.

Moreover, the correlation coefficients of the $\mathrm{PC}$ index and the AES-80 with the IMF $B_{z}$ component (figure not shown) are rather equivalent considering the whole one-year period May 1994-April 1995. These coefficients are respectively 0.37 and 0.4 all over the year considered, and these are above the $99.9 \%$ confidence level. It could be expected that both indices would have significant correlation with southward IMF (e.g., for PC, Friis-Christensen and Wilhjelm, 1975; Troshichev et al., 1988), but it is of interest to note the similarity in the correlations with each other. In fact, as written in the Introduction, the derivation of the two indices is done using different procedures of calculation which, in principle, refer to different components of the polar cap ionospheric 


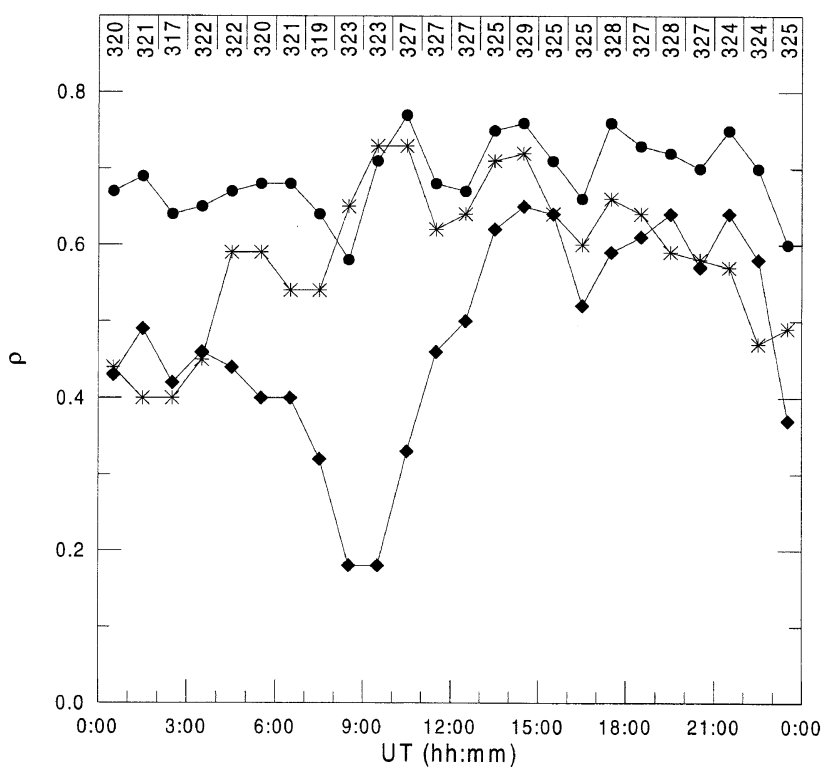

Fig. 5. Correlation coefficients for the PC index with AES-80 (solid circle), with ALS-80 (asterisk) and with AUS-80 (solid diamond) at different one-hour time intervals; the numbers at the top of the panel indicate the number of data points considered for each corresponding one-hour correlation.

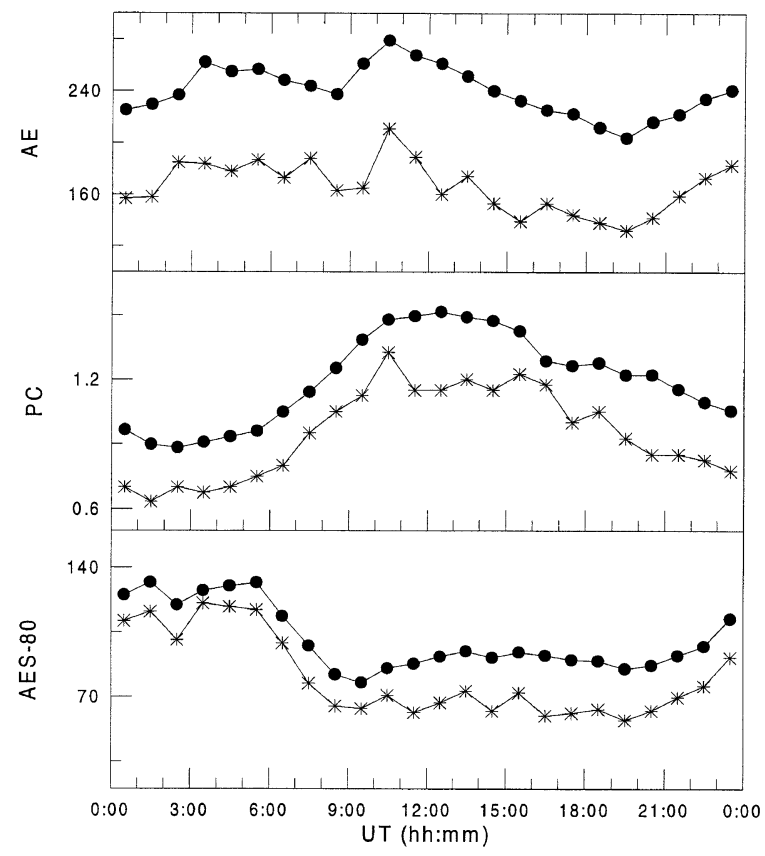

Fig. 6. Average (solid circle) and median (asterisks) of AE (top panel), PC (middle panel) and AES-80 (bottom panel) for separate UT hours. current system. The consideration of the correlation of AES80 and $\mathrm{PC}$ with the IMF $\mathrm{B}_{\mathrm{z}}$ during separate smaller periods can give higher correlation coefficients, as expected, which are sometimes slightly higher for one or for the other indices (Paper I). This variation in correlation could be due to seasonal effects, which will be addressed in a subsequent paper. For the purpose of comparison, the correlation coefficient between the AE index and IMF $B_{z}$ and it is 0.39 for the period May-December 1994 (the only presently available for AE index). We note that value is of the same order as the previous two. This comparison thus suggests that the dependence on southward IMF is similar in the auroral oval region and in the polar cap.

These results show that the effectiveness of AES- 80 as an indicator of the IMF status is as significant as for the other geomagnetic indices considered.

Acknowledgments. The authors thank L. J. Lanzerotti for useful comments and suggestions. Thanks are due to the Ecole et Observatoire de Physique du Globe, Strasbourg, France, and the Australian Antarctic Division, Hobart, Australia, for the magnetometer data from Dumont D'Urville and Casey, respectively; and $\mathrm{S}$. Vennerstrøm from the Danish Meteorological Institute for providing the official PC index from Thule. The magnetometers at McMurdo and the Automatic Geophysical Observatories are supported in part by the Office of Polar Programs of the U.S. National Science Foundation.

The Editor thanks T. Araki and other two referees for their assistance in evaluating this paper.

\section{References}

Allen, J. H. and H. W. Kroehl, Spatial and temporal distributions of magnetic effects of auroral electrojets as derived from AE indices, J. Geophys. Res., 80, 3667, 1975 .

Ballatore, P., C. G. Maclennan, M. J. Engebretson, M. Candidi, J. Bitterly, C. I. Meng, and G. Burns, A new southern high latitude index, Ann Geophysicae, 16, 1589, 1998.

Davis, T. N. and M. Sugiura, Auroral electrojet activity index AE and its universal time variations, J. Geophys. Res., 71, 785, 1966.

Friis-Christensen, E. and J. Wilhjelm, Polar cap currents for different directions of the interplanetary magnetic field in the Y-Z plane, J. Geophys. Res., 80, 1248-1260, 1975.

Saroso, S., Relationship between IMF By and geomagnetic activity in the southern polar cap, Ann. Geophysicae, 11, 470, 1993.

Saroso, S., M. Sugiura, T. Iyemori, T. Araki, and T. Kamei, Derivation of polar cap AE indices, Proc. NIPR Symp. Upper Atmos. Phys., 5, 35-45, 1992

Troshichev, O. A., V. G. Andrezen, S. Vennerstrøm, and E. Friis-Christensen, Magnetic activity in the polar cap-a new index, Planet. Space Sci., 36, 1988.

Vennerstrøm, S., E. Friis-Christensen, O. A. Troshichev, and V. G. Andrezen, Comparison between the polar cap index, pc, and auroral electrojet indices AE, AL, and AU, J. Geophys. Res., 96, 101, 1991.

Weatherwax, A. T., T. J. Rosenberg, C. G. Maclennan, and J. H. Doolittle, Substorm precipitation in the polar cap and associated Pc5 modulation, Geophys. Res. Lett., 24, 579, 1997.

P. Ballatore (e-mail: ballator@cnrs-orleans.fr) and C. G. Maclennan 